\title{
The Research and Development of the External Magnetic Field Acting on Electro-Deposition Process
}

\author{
Menghua $\mathrm{Wu}^{1}$, Weiping $\mathrm{Jia}^{2}$ \\ ${ }^{1}$ College of Mechanical Engineering, Dalian University, 116622, Dalian, China \\ ${ }^{2}$ Key Lab of Non-traditional Machining\& Functional Materials Preparing, Dalian University, 116622, Dalian, China
}

\begin{abstract}
The research and development status of the electro-deposition technology under the action of external magnetic field are introduced. The basic characteristics and applied manners of external magnetic field in electrodeposition process are summarized. The acting principle of external magnetic field, the effects of magnetic hydrodynamics (MHD) caused by the Lorentz force, and the acting of magnetic force on the metal ions and particles are described. The main actions of external magnetic field include MHD effect, magnetizing force, affecting the physical and chemical properties of the bath, affecting the disperse ability and coverage capacity of bath, affecting the mass transfer process of electro-deposition, affecting the chemical reaction process and current distribution of electrode surface. Some examples of electro-depositing single metal coatings, alloy coatings and composite coatings under action of magnetic field are explained. During the electro-depositing process, the external magnetic field has different degrees of impact on solution properties, mass transfer, charge transfer, content of composited nanoparticles, crystal growth and crystal orientation etc. The specific impact of magnetic field during the electro-depositing is also classified and summarized. The problems that existed in electro-deposition process while applying magnetic field and the next development trend were summarized.
\end{abstract}

\section{Introduction}

The electro-deposition preparation technology underaction of magnetic field is a integrated new technology which across the theory of magnetic field, electro-deposition processes and materials science areas, and it is gradually developing into an emerging interdisciplinary-Magnetic electrochemistry [1], [2]. As the magnetic fields posses many advantages, such as, high energy density and easy to be controlled, material is applied to the non-contact mode of action, so the thermal and kinetic energy may be transmitted to the material in the case of non-polluting, and so on. It has been concerned recently by the majority of researchers [3]. The magnetic field applied manners during electro-deposition process are mainly perpendicular to the current direction $(\mathrm{B} \perp \mathrm{J})$ and parallel $(\mathrm{B} / / \mathrm{J})$, and it may generate the Lorentz force, magnetic force, the magnetic hydrodynamic forces, etc. These forces have a certain impact on the mass transfer process, ion sports, bath state, surface state of coating, and the metal crystallization process, etc. So, it can improve the quality and performance of coatings [4]-[6]. In this paper, some research results of external magnetic field applied to electro-deposition are speech summed, the working principle and the specific role forms of the external magnetic field in the electro-deposition process are described, the problems in the application stage and further research trend of applying external magnetic field in electro-deposition process are clarified.

\section{Research overview of applying external magnetic field during the electro-deposition process}

Since the pioneering research work about acting of magnetic field on electrolyte solution was completed by Faraday in 1930s, as a new form of energy, the magnetic field reflect many other advantages, such as, improving the quality of prepared materials, saving energy, improve the environment, etc. While external magnetic field is applied in electro-deposition process, the magnetic hydrodynamics (MHD) effects generated by magnetic field may affect the physical and chemical properties, dispersion capacity and coverage ability of the bath, and also have an effect on the mass transfer and the deposition rate of charged particles, the current distribution on the electrode surface, the discharge and grain growth process, and then improve the morphology and mechanical or physical properties of coatings.

Presently, the research about actions of the magnetic field in electro-deposition process mainly focused on two aspects: The acting directions and the intensity of applied magnetic field [3], [7]. The types of electro-deposition coatings which prepared under the action of magnetic 
field are as follow: Single metal coatings, alloy coatings and composite coatings [8], [9].

\subsection{Electro-depositing single metal coatings under action of magnetic field}

The research and application of magnetic field applied to prepare single metal coatings are more extensive and mature. So far, the research about electro-deposition process under the action of magnetic field for preparing single metal coatings, such as nickel, iron, cobalt, chromium, zinc, tin, lead, copper, bismuth etc., has been carried out and achieved certain results. For example:

Kristina Tschulik et al [3]. conducted electrodeposition of separated 3D metallic structures by pulsereverse plating in magnetic gradient fields. Separate columns and stripes of $\mathrm{Cu}$ were generated by pulse reverse plating controlled by tailored magnetic field gradients. The experimental observations were explained by the action of the magnetic field gradient force on the paramagnetic $\mathrm{Cu} 2+$-ions during the deposition and the dissolution part of the cycle. Additional influences of the Lorentz force are also discussed.

Weiping Jia et al [10]. conducted research on surface morphology and texture of nickel crystal micro-casting by electro-forming under magnetic field. The results shown that the texture and surface morphology of the micro components are influenced by the current density and the magnetic field intensity. With the increase of current density, the size of crystal grains decrease firstly but increase later. And as the increase of the magnetic field intensity, the sizes of crystal grains become smaller and more uniform. Besides, the current density and the magnetic field intensity have a great impact on the texture of castings. As the increase of the current density, the texture orientation changes from (111) to (200). At the same time, along with the increase of the magnetic field intensity, the diffraction peak (200) is suppressed while the diffraction peak (111) is enhanced obviously.

\subsection{Electrodepositing alloy coatings under action of magnetic field}

How to change the composition ratio of alloy coating and improve its performance has become a hot field of electro-depositing alloy technique. If the external magnetic field is applied to electro-deposition process of alloy coatings, the characteristics of the external magnetic field could be taken full advantage for metal ion transmission and grain growth during the electrodepositing, so as to achieve the purpose of improving the coating properties. But it is not easy for electrodepositing alloy coatings to apply the external magnetic field due to the ferromagnetic, paramagnetic and diamagnetic of metal. So far, most relative research about electro-depositing alloy coatings under action of external magnetic field are focused on preparing nickel-iron or iron-nickel alloy coatings. For example:

Adriana Ispas et al [6]. conducted the study of external magnetic field effects on nickel-iron alloy electro-deposition based on linear and non-linear differential AC electrochemical response measurements. It was shown that by using linear electrochemical impedance spectrometry, it can be very difficult-if not almost impossible-to identify magnet hydrodynamic effects on the electro-deposition of $\mathrm{Ni}-\mathrm{Fe}$ alloys. It was shown that by using a non-linear approach the real and imaginary parts of the total impedance of the system are sensitive to the presence of a magnetic field in the frequency range from 1 to $100 \mathrm{~Hz}$.

Donggang Li et al [11]. conducted the evolution of morphology in electro-deposited nanocrystalline $\mathrm{Co}-\mathrm{Ni}$ films by in-situ high magnetic field application. The effect of high magnetic fields may up to $12 \mathrm{~T}$. Their research results shown that along with the increase of magnetic flux density, the grain size and the surface roughness of the films increased to reach a maximum value at a field of $9 \mathrm{~T}$. Meanwhile, higher magnetic flux density could improve cobalt atomic percentage in the film due to the impacts of magnet hydrodynamic effect. However, at a high field of $12 \mathrm{~T}$, the paramagnetic force played a predominant role in a decrease of mass transport, resulting in minimum grain size and roughness. The average values of surface roughness and lateral feature size, which can be used to characterize the grain size, were calculated using standard Nova software of AFM.

Marek Zieliński [12] presented a study of the effect of constant magnetic field (CMF) on the basic processes of cobalt-tungsten alloys electro-deposition. The exposure to CMF caused also an increase of the volume fraction (by about $9 \%$ by volume) of the dominant phase (Co3W and Co7W6) in the alloy. The reason for these changes was the fact that the Lorentz force, generated in CMF, caused the magnetic hydrodynamic (MHD) effect. This induced movement of the electrolyte. The Nernst diffusion layer was depleted, whereas a new Naviere Stokes hydrodynamic layer appeared, which determined the velocity of electro active molecules flow to the working electrode under CMF conditions.

P. Żabiński et al [7]. conducted the research on electro-catalytically active $\mathrm{Co}-\mathrm{W}$ and $\mathrm{Co}-\mathrm{W}-\mathrm{C}$ alloys electro-deposited in a magnetic field. The influence of sodium tungstate concentration, arginine concentration and orientation of applied magnetic field (MF) on composition, morphology, structure, cathodic current efficiency of deposition process and electro-catalytic activity of deposited alloys were examined. The positive effect of carbon addition on catalytic activity of hydrogen evolution was observed. The influence of electrodeposition parameters on size of micro cracks formed during deposition processes was also investigated.

\subsection{Electro-depositing composite coatings under action of external magnetic field}

The content of nanoparticles in composite coating is a key factor to determine the composite coating performance, and how to improve the content of nanoparticles in the composite coating has become a recently hot research. When external magnetic field is introduced into electro-depositing process, it may not only produce a beneficial stirring to the bath, let 
nanoparticles uniformly and stably suspended in the bath, but also accelerate the transmission process of particles, so as to provide effective way of solving agglomeration of the nanoparticles. Also, the content of nanoparticles in composite coatings may be increased to a certain extent, thereby the performance of the composite coatings may be greatly improved. For example:

Pengwei Zhou et al [2], [13]. conducted the research on behavior of Fe/nano-Si particles composite electrodeposition with a vertical electrode system in a static parallel magnetic field. Compared to the rather smooth surface morphology of Fe-nano Si composite coating without magnetic field, it became wavy and striped obviously with imposing magnetic field. The nano silicon particles dispersed uniformly on the surfaceof coating when no magnetic field was applied, whereas the nano silicon particles content of coatings presented a trend of increasing with increasing the magnetic flux density. The influence of an external parallel strong parallel magnetic field (respect to current) on the electro-deposition of nano-silicon particles into an iron matrix had been studied in their another paper. Test results show that magnetic field has a great influence on the distribution of silicon, as well as the surface morphology and the thickness of the composite coatings. The thicker and rougher composite deposits appearin the edge region (Lor $\mathrm{R}$ region), and the thinner and smoother ones appear in the middle region (M). The distribution curve of silicon content looks like a "pan" along the centre line of coatings.

Denny Thiemig et al [14]. studied the effect of an external magnetic field on the electro-deposition of composites consisting of either $\mathrm{Co}$ or magnetite nanoparticles in a Ni matrix. The particle incorporation showed a distinct dependency on the orientation of an externally applied magnetic field. While the particle incorporation increased in a perpendicular field (perpendicular with regard to the electrode surface), it decreased in a parallel orientation. This result was explained with the dominating action of the magnet phoretic force. The structure and the properties of the $\mathrm{Ni}$ layers were significantly affected by the particles codeposition. A refinement of the Ni grains was found with increasing plating current density and as a result of the nanoparticle incorporation. The magnetic hardness and the Vickers micro hardness of the films increased significantly due to the incorporation of the nanoparticles.

Chao Wang et al [15]. applied an external high parallel magnetic field into the co-deposition process of $\mathrm{Ni} /$ nano-A12O3 composites. It was found that the distribution of nanoparticles showed the network shape when super imposing a magnetic field, and the average size of the single network increased with increasing the current density. High concentration of nanoparticles could be obtained at low current density with a magnetic field, while a high current density was needed to realize that when without a magnetic field. The super imposed magnetic filed would improve the current efficiency.

\section{The working principle of external magnetic field}

The magnetic field is produced by moving charges or changing electric field. The basic characteristics of magnetic field is that it is able to apply force to the electric charges which moving in magnetic field, i.e., the energized conductor is affected by the magnetic force in magnetic field [16]. When magnetic field was applied in the material preparation process, the electromagnetic force acting on the prepared material may be expressed as follow equation [17], [18]:

$$
F=j \times B+\rho_{e} E+\left(\mu_{0} M \cdot \nabla\right) H+(P \cdot \nabla) E
$$

In the right side of above equation, the first term is the Lorentz force, the second term is the Coulomb force, the third term is the magnetizing force, and the fourth term is the polarization force. The main forces which ions or materials subjected to in magnetic field are Lorentz force and magnetic force, and the two main forces produced by magnetic field are normally used to improve the preparing process of materials. When the magnetic field strength reaches a certain value (a few $\mathrm{T}$ to dozens $\mathrm{T}$ ), it can even have an effect of Lorentz force and magnetic force on a very weak current and non-magnetic materials, thus largely expand its scope of application. Influence of magnetic field on material preparation process may performance in many ways: distribution of particles in composite coatings may be affected by the Lorentz force of the magnetic field, mass transfer process and crystal orientation may be affected by magnetizing force, in addition, chemistry reaction also may be affected to some extent by the magnetic field [19], [20]. Under acting of $10 \mathrm{~T}-20 \mathrm{~T}$, even $100 \mathrm{~T}$ super magnetic field, there are very significant influences on the chemical reaction system, and thus indirectly affect the performances of prepared materials.

With the development of super conducting technology, it will be more easy to obtain strong magnetic field. Super conducting magnets with 10T magnetic field have been commercially available in the international, so the non-magnetic material can easily be magnetized, and so as to the possibility of using the method of materials preparation to access to new theories and discoveries have also been increased [21], [22].

\section{The action of external magnetic field on electro-deposition process}

Normally, the electro-deposition process can be roughly divided into the following steps: Mass transfer, electrochemical and electrical crystallization [23], [24]. If the magnetic fields are applied during the electrodeposition process, it would have a corresponding effect on the steps mentioned above, and ultimately affect the morphology and properties of the coatings. These effects embodied in the following aspects [7], [12]:

The Magnetic Hydro Dynamic (MHD) effect caused by Lorentz force [9], [17]. The Lorentz force caused by Interaction of magnetic fields and current may generate the macroscopic MHD effects and micro-MHD effects, these effects not only enhance the transmission of mass, but also play the role of stirring the solution and make the solution more uniform, thereby improving the quality of 
the coatings. In the electro-deposition process, the macroscopic MHD effects perform the action of Lorentz force when plasma flow cutting magnetic lines. The result is that the bath is stirred, and this effect can be observed when applying relatively weak magnetic field. When applying strong magnetic field, the MHD effect will act on the trajectory of each ion, and the Lorentz force will also act on the micro current between the oxidation particles and reduction particles. This phenomenon is called micro-MHD effect [12]. When applying the vertical magnetic field, the electric force and the Lorentz force act at the same time on the charged particles in solution, and the charged particles will perform a spiral movement, then disturbance the solution to form a MHD effect. Finally, it will result changing of solution performance and variation of grain size in depositing layer.

Action of magnetizing force. Atomic electrons carried spin motion on the one hand, and kept movement around the nucleus on the other hand. Electrons can be equivalent to this feature as a small ring current, called molecular currents [8]. In the absence of external magnetic field, the orientation of these molecular current is random and does not exhibit any macroscopic effects. Once the magnetic field was applied, these molecular current appear regular orientation and form macroscopic magnetization current. This phenomenon is called the magnetic substance. When the applied magnetic field is parallel to the current manner during the electrodeposition process, with no Lorentz force, the magnetization force will become very apparent. Thus, the metal ions and particles will be subject to the ferromagnetic matrix metal and be easy to deposit with lowest energy state on the surface of the base body during depositing process. So that the coating is more compact with fewer defects [9]. The intrinsic reasons that magnetizing force may impact on the electro-deposition process are as follow: Coating crystal exists magnetic anisotropy, there are differences in the magnetic energy of the various ion in bath.

Affecting the physical and chemical properties of the bath. The magnetic field may cause the physical properties of water change, such as, reducing the surface tension, improving wettability, altering the process of dissolution, crystallization and condensation, etc [25], [26]. Since the majority of the bath is aqueous system, so applying magnetic field may accordingly impact the bath solubility, viscosity, wettability and liquidity, and furthermore impact the electro-deposition process.

Affecting the disperse ability and coverage capacity of bath. The MHD effect generated by the magnetic field can accelerate the mass transfer process, make the viscosity coefficient of bath be smaller, enhance conductivity and cathodic polarization, thereby improving the disperse ability of the bath. The electric line distribution at concave surface of the coating tends to be uniform under the action of magnetic field. Ion trajectory will be changed while the ions in bath are affected both by electric and magnetic forces, and the shielding effect of protrusions surface will be weakened, so that coverage capacity of bath is improved [6], [10].
Affecting the mass transfer process of electrodeposition. The electrode process of electro-deposition involves liquid mass transfer process and the electrode reaction process. The liquid mass transfer process include three transfer forms: Convection, diffusion and electromigration. These three mass transfer processes always occur simultaneously. However, under certain circumstances, there are normally one or two transfer forms play a major role. After applying a magnetic field, the magnetic induced convection effect which generated by Lorentz force will act on the bath ions and cause convection diffusion, thereby thinning the thickness of the diffusion layer and accelerating the mass transfer process [6], [20]. Special attention is required because the magnetic field not only affect the mass transfer rate but also affect the charge transfer [8].

Affecting the chemical reaction process. Magnetic field can not only control the macro physical and chemical reactions of material, but also influence and regulate the inside microscopic state of substances [9]. Magnetic field may affect chemical reaction heat, $\mathrm{pH}$ value, direction of chemical reaction, reaction rate, activation energy, entropy and many other factors, thereby affecting the electro-deposition process and coating performance.

Affecting the current distribution of electrode surface. Under the action of magnetic field, the polarization will increase where the current density of cathode surface are larger [11]. The increasing polarization, in turn, contribute to the current distribution of cathode surface tends to uniform, thereby improving the coating uniformity on electrode surface. Up to now, some research achievements of applying magnetic field in the field of electro-deposition have been gained. Scholars from various countries have experimentally studied the electro-deposition process under the action of different magnetic field strength and different acting directions, and the acting mechanism of magnetic field on electrodeposition process have been carried out more in-depth discussion and analysis [4], [27], [28]. Learned that the MHD effect and action of magnetizing force generated by the magnetic fluid effect were the major factors which can affect the process of electro-deposition, and the affect mechanism of each factor on electro-deposition process were specifically analyzed. The MHD effect is macroeconomic performance of Lorentz force generated by perpendicular magnetic field, it may accelerate the mass transfer process and improve the disperse ability of bath by stirring the bath, and then improve the performances of coatings [25, 29]. The functions of parallel magnetic field are mainly performance the effect of magnetizing force, this effect may have a significant impact on transporting of particles, the chemical reaction rate, charge transfer, and the crystal growth process, thereby affecting the morphology, tissue and properties of coating [27], [28].

\section{The problems of applying external magnetic field during the electro- deposition process}


Although the research work about applying magnetic field on electro-deposition process has been made some progress, and to some extent, has been applied. However, according to the situation of the current research, the research work still exist some problems and shortcomings:

Firstly, the affecting mechanism of the magnetic field on electro-deposition process is now more clearly that the MHD effect and the magnetic force effect generated by the magnetic field on the electro-deposition process. However, it is not very clear about the variation laws of physical and chemical properties of the bath and electrochemical reaction rate.

Secondly, the current experiments are mostly simple magnetic field effect on the electro-deposition process, but the composite experimental studies of magnetic field and other physical fields (such as ultrasonic wave, flow field generated by mechanical stirring, etc.) have not been fully conducted, and it is unclear about the composite affecting mechanism of multi-physical fields. Therefore, the research depth of which should be extended.

Thirdly, there are relatively more research about affecting of weak magnetic field on the electro-deposition process, and relevant theories are more mature, but there are less research or just get starting about affecting of strong magnetic fields (10T or more) on the electrodeposition process. Strong magnetic field will not only have an magnetization effect on ferromagnetic material, but also have an magnetization effect on the diamagnetic and paramagnetic materials, so the electro-deposition process under acting of strong magnetic field will become more complex. Therefore, it is urgent to prove the acting mechanism of strong magnetic field on electro-deposition process and performance of coatings.

Fourthly, there are relatively more experimental study of preparing magnetic materials and anisotropic materials by applying magnetic field. However, it is unclear about the affecting laws of strong magnetic fields on the mass transfer process of ferromagnetic ions and particles in bath, and on the nucleation and growth pattern of grains. So the experimental study in this regard should be strengthened.

Fifthly, since the metal itself has a ferromagnetic, paramagnetic and diamagnetic, the electro-depositing alloy coating process under action of magnetic field will become complicated. Thus the experiments of electrodepositing different types of alloy coating under action of magnetic field will be limited and constrained.

In short, there is lot of research space for applying the magnetic field in the field of electro-deposition technology. Therefore, to carry out further research and exploration is very necessary.

\section{Conclusions}

Combining the current research status and problems about applying magnetic field in the electro-deposition process, the next research trends in this field be summarized as follows:

a) In order to more comprehensive and in-depth grasp the influence of magnetic field on the electro-deposition process, the research about acting mechanism of magnetic field on the performance of bath, the electrochemical reaction rate, the crystal growth process, the plasma discharge process and other aspects should be strengthened.

b) The compound electro-deposition experiments and research, which combined the action of magnetic field and other fields (such as ultrasonic wave, flow field electric field, etc.), should be conducted for proving the complex acting mechanism of multi-physics fields applied in electro-deposition process.

c) As strong magnetic field may have magnetization to the non-magnetic materials, the experimental study of electro-deposition under the action of strong magnetic field should be conducted, so as to master and apply the electro-deposition process under the action of strong magnetic field.

d) The further study of electro-depositing alloy process under the action of the magnetic field should be conducted, so as to investigate the effect of magnetic field on the composition and properties of alloy coating.

e) As the substrates for electro-deposition are diamagnetic material or ferromagnetic material, so it is necessary to compare the action of magnetic field on the different substrate bath.

\section{Acknowledgements}

This work was supported by the Dalian Science and Technology Project (2015A11GX003) and the Science \&Technology Research Project of Education Department of Liaoning province (L2015029).

\section{References}

1. K. Tschulik, J. A. Koza, M. Uhlemann, Electrochemistry Communications. J. 11(2009), p.2241-2244

2. Pengwei Zhou, Yunbo Zhong, Huai Wang, Lijun Fan, Electrochimica Acta. J. 111 (2013), p. 126-135

3. Kristina Tschulik, Ralph Sueptitz, Margitta Uhlemann, Electrochimica Acta. J. 14 (2011), p. 5174-5177

4. Yanling Wen, Yunbo Zhong, Zhongming Ren, The Chinese Journal of Nonferrous Metals. 4 (2006), p. 715-720

5. Yundan $\mathrm{Yu}$, Lixia Sun, Junwei Lou, Hongliang Ge, Electroplating \& Pollution Control. J. 4 (2012), p. 13 "In Chinese"

6. Adriana Ispas, Hisayoshi Matsushima, Andreas Bund, Electro-analytical Chemistry. J. 2 (2011), p.197-203

7. P. Żabiński, K. Mech, R. Kowalik, Electrochimica Acta. J. 1 (2013), p. 542-548

8. Chi-Cheng Hung, Wen-Hsi Lee, Japanese Journal of Applied Physics. 48 (2009), p.076506-1

9. Mehdi Ebadi, W. J. Basirun, Yatimah Alias, Journal of Chemical Sciences. 2 (2010), p.279-285

10. Weiping Jia, Fan Yang, Menghua Wu, Advanced Materials Research. J. 535-537 (2012), p.450-454

11. Donggang Li, Alexandra Levesque, Agnieszka Franczak, Talanta. J. 110 (2013), p. 66-70 
12. Marek Zieliński, Materials Chemistry and Physics. J. 1 (2013), p.370-377

13. Pengwei Zhou, Yunbo Zhong, Huai Wang, Qiong Long, Applied Surface Science. J. 282(2013), p. 624-631

14. Denny Thiemig, Clemens Kubeil, Christian P. Gräf, Andreas Bund, Thin Solid Films. J. 517 (2009), p.1636-1644

15. Chao Wang, Yunbo Zhong, Weili Ren, Zuosheng Lei, Applied Surface Science. J. 254 (2008), p.5649-5654

16. W.Szmaja, W. Kozowski, K. Polanski, J. Balcerski, Mater. Chem. Phys. J. 132 (2012), p.1060-1066

17. Nana Liu, Menghua Wu, Zhi Li, Yuangang Wang, Journal of Rare Metal Materials \& Engineering. 3 (2013), p. 649-655 "In Chinese"

18. S.A. Lajevardi, T.Shahrabi, J.A. Szpunar, Applied Surface Science. J. 279(2013), p.180-188

19. O. Ergeneman, K.M. Sivaraman, S. Pane, E. Pellicer, Electrochimica Acta. J. 56 (2011), p.1399-1408

20. Shuhong Wang, Huanping Zhang, Hui Du, Shaogang Hou, Yong Zheng, Anlin Li, Applied Chemical Industry. J. 44 (2015), p.732-735 "In Chinese"
21. P. Żabiński, K. Mech, R. Kowalik, Archives of Metallurgy and Materials. J .57 (2012), p. 127-133

22. Xiao-qin Ouyang, Linyan Zhou, Bin Yu, Ying Wan, Bin-bin Zhang, Shuilian Hu, Changjie Feng, Plating and Finishing, J. 36 (2014), p.20-29 "In Chinese"

23. Jiao Feng, Nanjing University of Aeronautics and Astronautics, D, (2009). "In Chinese"

24. F. C. Walsh*, and C. Ponce de Leon, Transactions of the IMF. 2 ( 2014), p. 84-98

25. C. Wang, Y. B. Zhong, J. Wang, Journal of Electroanalytical Chemistry. 1-2 (2009), p.42-48

26. Lorena M.A. Monzon, J.M.D. Coey, Electrochemistry Communications. J. 42 (2014), p. 38-41

27. Peng Gao, Zhongdong Yang, Xiaolian $\mathrm{Xu}$, Transaction of Materials \& Heat treatment. J. 4 (2012), p.136-140 "In Chinese"

28. Jianming Lu, David Dreisinger, Thomas Glück, Hydrometallurgy. J. 141 (2014), p.105-116

29. Yundan Yu, Lixia Sun, Junwei Lou, Hongliang Ge, Electroplating \& Pollution Control. J. 4 (2012), p. 13 "In Chinese" 